\title{
Metformin or gliclazide, rather than glibenclamide, attenuate progression of carotid intima-media thickness in subjects with type 2 diabetes
}

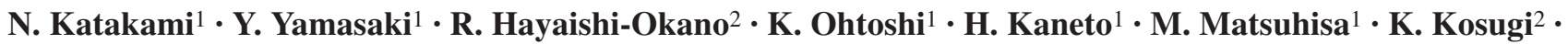 \\ M. Hori ${ }^{1}$ \\ ${ }^{1}$ Department of Internal Medicine and Therapeutics, Osaka University Graduate School of Medicine, Suita City, Osaka, Japan \\ 2 Osaka Police Hospital, Osaka, Japan
}

\section{Abstract}

Aim/hypothesis. Metformin is a well-known oral hypoglycaemic agent and has been commonly used, in combination with sulphonylurea, to treat type 2 diabetes. However, the advantageous effect of metformin plus sulphonylurea on diabetic macroangiopathy has yet to be clarified. To evaluate whether sulphonylurea or sulphonylurea plus metformin prevent diabetic macroangiopathy, we examined the progression of carotid artery intima-media thickness (IMT) as a surrogate end point.

Methods. Subjects with type 2 diabetes were divided into three groups, receiving the following treatments: (i) glibenclamide $(n=59)$; (ii) gliclazide $(n=30)$; and (iii) glibenclamide + metformin $(n=29)$. Maximum IMT and average IMT (the greatest value among 6 average values of each 3 points including greatest thickness) were measured at the beginning and end of the observation period.

Results. For the follow-up period of 3 years, the annual change in average IMT of the glibenclamide plus metformin group $(0.003 \pm 0.048 \mathrm{~mm})$ was smaller than that of the glibenclamide group $(0.064 \pm 0.045 \mathrm{~mm})$ and gliclazide group $(0.032 \pm 0.036 \mathrm{~mm})(p<0.0001$ and $p=0.043$ respectively). In the gliclazide group, average IMT increased during the follow-up period, but annual change in average IMT was significantly smaller than that of the glibenclamide group $(p=0.005)$. Glibenclamide + metformin or gliclazide also attenuated the progression of maximum IMT, compared with that of glibenclamide $(0.041 \pm 0.105$, $0.044 \pm 0.106,0.114 \pm 0.131 \mathrm{~mm} /$ year respectively, $p=0.029$ and $p=0.035$ respectively). Multivariable regression analysis implied that administration of metformin or gliclazide significantly and independently $(p<0.05)$ reduces the progression of average IMT, compared with glibenclamide monotherapy.

Conclusions/interpretation. These data indicate that metformin or gliclazide, rather than glibenclamide, have a potent anti-atherogenic effect in type 2 diabetes.

Keywords Atherosclerosis - Carotid · Follow-up study · IMT · Metformin · Multivariable analysis · Sulphonylurea $\cdot$ Surrogate end point
Received: 18 March 2004 / Accepted: 13 July 2004

Published online: 24 November 2004

C) Springer-Verlag 2004

Y. Yamasaki $(\bullet)$

Department of Internal Medicine and Therapeutics, Osaka University Graduate School of Medicine,

2-2 Yamadaoka, Suita City, Osaka 565-0871, Japan

E-mail: yamasaki@medone.med.osaka-u.ac.jp

Tel.: +81-6-68793633, Fax: +81-6-68793639

Part of this study was reported as a poster presentation at the EASD annual meeting, held in Budapest in 2002

\section{Introduction}

The effect of intensive blood glucose control with oral hypoglycaemic drugs on complications in patients with type 2 diabetes was evaluated in the UK Prospective Diabetes Study (UKPDS). In UKPDS [1], metformin monotherapy was associated with a significant decrease in the risk of any diabetes-related end point,

Abbreviations: IMT, intima-media thickness ·

UKPDS, UK Prospective Diabetes Study 
diabetes-related death, all-cause mortality, and myocardial infarction. Metformin also had a greater beneficial effect than chlorpropamide, glibenclamide, or insulin for any diabetes-related end point, all-cause mortality, and stroke [1]. However, these results have been questioned, because patients with poorly controlled type 2 diabetes who had been treated with sulphonylurea appeared to have an increased risk of diabetes-related death and death from any cause when metformin was given in addition to sulphonylurea. Thus, the effect of combined metformin and sulphonylurea treatment on diabetic macrovascular complications in patients previously treated with sulphonylurea had not been determined and required further study.

The increased oxidative stress and glycation seen in diabetes are fundamental to the pathogenesis of diabetic macrovascular disease [2]. Since gliclazide not only lowers blood glucose, but has also been shown to be a free radical scavenger [3], it may be more effective than other sulphonylureas in preventing macrovascular complications. Indeed, some studies have shown that gliclazide has beneficial effects on the haemorrheological abnormalities seen in diabetic vascular disease $[4,5,6,7,8,9]$. However, it has not yet been proved that gliclazide really decreases the risk of macrovascular complications.

Increased intima-media thickness (IMT) of the carotid artery is considered to be an index of the progression of atherosclerosis and a useful predictor of a high-risk group of cardiovascular events $[10,11,12$, $13,14,15]$. To evaluate whether the drugs named above have potent anti-atherogenic effects on diabetic patients, we did an open randomised prospective study examining the progression of carotid artery IMT as a surrogate end point.

\section{Subjects and methods}

Subjects. Subjects were selected from outpatients attending Osaka University Hospital and Osaka Police Hospital as follows. All type 2 diabetic patients who visited the hospitals during 2 months (May-June, 1998) and had been treated with glibenclamide (1.25-7.5 mg/day, $n=100)$, gliclazide $(20-120 \mathrm{mg} /$ day, $n=45)$ or glibenclamide + metformin $(1.25-5 \mathrm{mg} /$ day and $500-750 \mathrm{mg} /$ day respectively, $n=46$ ) were asked to participate in the study. Patients suffering from advanced diabetic complications, severe renal or hepatic disease, apparent cardiovascular disease or malignancy were excluded. In the end, 81 (glibenclamide monotherapy group), 37 (gliclazide monotherapy group) and 41 sujects (glibenclamide + metformin group) met the criteria and attended the study. None of these patients was taking other hypoglycaemic drugs or anti-platelet drugs during the observation period. The other drugs administered (including hypolipidaemic drugs and anti-hypertensive drugs) remained unchanged during the observation period. The diagnosis of type 2 diabetes was based on World Health Organization criteria. The hospital ethics committee approved the study protocol, and informed consent was obtained from each patient.
Acquisition of data. Ultrasonographic scanning of the carotid artery was performed at the beginning and the end of the study and measurements of other parameters were repeatedly performed (every 2-3 months) during the observation period of $3.3 \pm 0.2$ years. A series of ultrasonographic scans of the carotid artery was performed using an echotomographic system (EUB-450, Hitachi Medico, Tokyo, Japan) with an electrical linear transducer (mid frequency of $7.5 \mathrm{MHz}$ ). All images were photographed. The detection limit of this system using 7.5 $\mathrm{MHz}$ was approximately $0.1 \mathrm{~mm}$. The conventional Bmode imaging of the extracranial common carotid artery, the carotid bulb, and the internal carotid artery in the neck was performed bilaterally from three different longitudinal projections (i.e. anterior-oblique, lateral and posterior-oblique), as well as from the transverse projection, as reported previously $[16,17]$. The carotid IMT defined by Pignoli and co-workers was measured as the distance from the leading edge of the first echogenic line to the leading edge of the second echogenic line [18]. At each longitudinal projection, the site of the greatest thickness including a plaque lesion was sought along the near and far arterial walls from the common carotid artery to the internal artery. We did three measurements of IMT at the site of the greatest thickness and at two other points located $1 \mathrm{~cm}$ upstream and $1 \mathrm{~cm}$ downstream of this site. When either of these sites (1 cm upstream/downstream) could not be measured, we measured the site $2 \mathrm{~cm}$ downstream (upstream) instead. These three measurements were averaged. The greatest value among 6 averaged IMTs ( 3 from the left and 3 from the right) was used as the representative average IMT for each individual. The greatest thickness among six longitudinal projections was used as the maximum IMT. Two physicians conducted all scans and another physician performed IMT measurements on the photographs. They were unaware of the clinical characteristics of the subjects. The reproducibility of the IMT measurements was examined by conducting another scan 1 week later on 8 subjects. The mean difference in IMT between these two measurements was $0.01 \mathrm{~mm}$ and the standard deviation was $0.04 \mathrm{~mm}$, demonstrating good reproducibility of repeated measurements. The annual changes in average IMT and maximum IMT were calculated using the following equations: (i) annual change in average IMT = (last average IMT-initial average IMT)/observation period; (ii) annual change in maximum IMT = (last maximum IMT-initial maximum IMT)/observation period.

Assessment of other parameters. Various laboratory data and blood pressure were measured at least twice a year during the observation period and averaged. Fasting blood was withdrawn for analysis of serum total cholesterol, serum HDL-cholesterol, serum triglycerides, plasma glucose and glycated haemoglobin $\left(\mathrm{HbA}_{1} \mathrm{c}\right)$ levels by standard laboratory techniques. Blood pressure was measured with a mercury sphygmomanometer. After a supine rest of $5 \mathrm{~min}$, three measurements in the sitting position were conducted, and the mean value was used. Body mass index of subjects was measured at the beginning and the end of the observation period. Patients' smoking status was classified as: never having smoked; former smoker (ceased smoking for at least 3 years); or current smoker. In this study, current smokers at the termination of the follow-up period were analysed as a group and compared with those who had never smoked and former smokers.

Antihypertensive drugs (diuretics, beta-blockers, alphablockers, Ca-channel blockers, and angiotensin-converting enzyme inhibitors) were given to 52 of the patients. Anti-hyperlipidaemic drugs were given to 48 of the patients (clofibrates to 6 subjects, probucol to 20 subjects, HMG-CoA reductase inhibitors to 29 subjects). The administration of these drugs re- 
Table 1. Patient characteristics before and after the follow-up period

\begin{tabular}{|c|c|c|c|c|c|c|}
\hline & \multicolumn{2}{|c|}{ Glibenclamide, $(n=59)$} & \multicolumn{2}{|c|}{ Gliclazide, $(n=30)$} & \multicolumn{2}{|c|}{ Glibenclamide+Metformin, $(n=29)$} \\
\hline & Before & After & Before & After & Before & After \\
\hline Age (years) & $60.3 \pm 10.3$ & & $60.8 \pm 6.6$ & & $62.8 \pm 8.9$ & \\
\hline Duration (years) & $13.3 \pm 9.8$ & & $13.4 \pm 8.5$ & & $14.8 \pm 10.3$ & \\
\hline BMI $\left(\mathrm{kg} / \mathrm{m}^{2}\right)$ & $23.2 \pm 3.1$ & $23.2 \pm 3.1$ & $22.9 \pm 3.2$ & $22.9 \pm 2.6$ & $22.9 \pm 2.6$ & $22.7 \pm 2.6$ \\
\hline $\mathrm{TG}(\mathrm{mmol} / \mathrm{l})$ & $1.55 \pm 0.99$ & $1.55 \pm 0.70$ & $1.56 \pm 0.91$ & $1.74 \pm 1.32$ & $1.75 \pm 1.34$ & $1.56 \pm 0.79$ \\
\hline HDL-chol (mmol/l) & $1.39 \pm 0.39$ & $1.30 \pm 0.35^{\mathrm{a}}$ & $1.48 \pm 0.49$ & $1.43 \pm 0.52$ & $1.42 \pm 0.27$ & $1.47 \pm 0.35$ \\
\hline $\mathrm{sBP}(\mathrm{mmHg})$ & $129 \pm 14$ & $137 \pm 15^{\mathrm{a}}$ & $133 \pm 16$ & $137 \pm 16$ & $133 \pm 13$ & $138 \pm 12^{\mathrm{a}}$ \\
\hline $\mathrm{dBP}(\mathrm{mmHg})$ & $75 \pm 9$ & $77 \pm 9$ & $77 \pm 9$ & $76 \pm 7^{a}$ & $76 \pm 8$ & $77 \pm 7$ \\
\hline AveIMT (mm) & $1.23 \pm 0.31$ & $1.43 \pm 0.35^{\mathrm{a}}$ & $1.25 \pm 0.31$ & $1.25 \pm 0.31$ & $1.33 \pm 0.33$ & $1.34 \pm 0.34$ \\
\hline MaxIMT (mm) & $1.59 \pm 0.66$ & $1.95 \pm 0.69^{a}$ & $1.66 \pm 0.63$ & $1.83 \pm 0.64$ & $1.78 \pm 0.62$ & $1.89 \pm 0.73$ \\
\hline
\end{tabular}

Data are shown as means \pm SD. Statistical significance was determined using the paired $t$ test. ${ }^{a} p<0.05$ vs values before treatment. There were no significant difference among the groups.
T-chol, total cholesterol; TG, triglyceride; HDL-chol, HDL cholesterol; sBP, systolic blood pressure; dBP, diastolic blood pressure; AveIMT, average IMT; MaxIMT, maximum IMT mained constant during the follow-up period. None of the patients was receiving anti-platelet drugs, insulin sensitisers (troglitazone or pioglitazone) or biguanides during the followup period. No patient showed any onset of coronary heart disease, cerebro-vascular disease or arteriosclerosis obliterans during the follow-up period.

Statistical analysis. Values are presented as means \pm SD and were compared by Student's and paired $t$ test or one-way analysis of variance. To account for the effects and interactions of different variables on the annual change in average IMT or the annual change in maximum IMT, stepwise multivariable regression analysis was performed. In this analysis, $F$ values for inclusion and exclusion of variables were set at 4.0. These statistical analyses were carried out using the HALBAU statistical package (Gendai Sugakusha, Kyoto, Japan) on a personal computer. A $p$ value of less than 0.05 was taken to be statistically significant.

\section{Results}

Overall parameters. During the follow-up period, 41 patients dropped out of the study. A total of 32 patients changed their initial drug regimens due to (i) marked worsening of glycaemic control or progression of diabetic complications $(n=25)$ or (ii) worsening of any other disease $(n=7)$. Attendance was interrupted by 9 patients, due to a change of domicile or unknown reasons. There were no CVD events during the follow-up period. In the end, the statistical analysis included the following numbers of type 2 diabetic subjects: treated with glibenclamide $(n=59)$; treated with gliclazide $(n=30)$; treated with glibenclamide + metformin $(n=29)$.

Effect of sulphonylurea and metformin on the progression of carotid artery IMT. Patient characteristics be- fore and after the follow-up period are shown in Table 1 . At the beginning of the observation period, the glibenclamide group, gliclazide group, and glibenclamide plus metformin group showed no significant differences in baseline average IMT $(1.23 \pm 0.31 \mathrm{~mm}$, $1.25 \pm 0.31 \mathrm{~mm}, 1.33 \pm 0.33 \mathrm{~mm}$ respectively) and maximum IMT $(1.59 \pm 0.66 \mathrm{~mm}, 1.66 \pm 0.63 \mathrm{~mm}$, $1.78 \pm 0.62 \mathrm{~mm}$ respectively). There were also no significant differences in the other clinical parameters such as body mass index, $\mathrm{HbA}_{1} \mathrm{c}$, lipid profile, and blood pressure (Table 1). At the end of the observation period, average IMT in both the glibenclamide group and gliclazide group $(1.43 \pm 0.35 \mathrm{~mm}$ and $1.36 \pm$ $0.31 \mathrm{~mm}$ respectively) significantly increased as compared with the baseline values. However, there was no significant increase between baseline average IMT $(1.33 \pm 0.33 \mathrm{~mm})$ and the last average IMT (1.34 \pm $0.34 \mathrm{~mm}$ ) in the glibenclamide plus metformin group. Maximum IMT also significantly increased as compared with the baseline in all groups. With regard to $\mathrm{HbA}_{1} \mathrm{c}$, a significant improvement was seen in both the gliclazide and glibenclamide plus metformin groups. In the glibenclamide group, total cholesterol and HDL-cholesterol concentrations significantly decreased during the follow-up period $(p<0.05$ for all significant values given above).

However, the average values of these parameters during the observation period showed no significant differences among the three groups (Table 2). The annual change in average IMT of the glibenclamide plus metformin group $(0.003 \pm 0.048 \mathrm{~mm})$ was significantly smaller than that of the glibenclamide group $(0.064 \pm 0.045 \mathrm{~mm})$ and gliclazide group $(0.032 \pm 0.036 \mathrm{~mm})(p<0.0001$ and $p=0.043$ respectively). In the gliclazide group, average IMT increased during the follow-up period, but the annual 
Table 2. Average of parameters during the observation period

\begin{tabular}{lccc}
\hline & Glibenclamide $(n=59)$ & Gliclazide $(n=30)$ & Glibenclamide + metformin $(n=29)$ \\
\hline BMI $\left(\mathrm{kg} / \mathrm{m}^{2}\right)$ & $22.8 \pm 3.6$ & $22.2 \pm 4.0$ & $22.8 \pm 2.5$ \\
$\mathrm{HbA}$ c $(\%)$ & $8.7 \pm 1.1$ & $8.1 \pm 1.1$ & $8.3 \pm 1.6$ \\
T-chol (mmol/l) & $5.45 \pm 0.79$ & $5.33 \pm 0.83$ & $5.14 \pm 1.03$ \\
TG (mmol/l) & $1.54 \pm 0.69$ & $1.63 \pm 1.06$ & $1.68 \pm 0.99$ \\
HDL-chol (mmol/l) & $1.31 \pm 0.34$ & $1.48 \pm 0.33$ & $137 \pm 14$ \\
sBP (mmHg) & $133 \pm 15$ & $134 \pm 15$ & $77 \pm 7$ \\
dBP (mmHg) & $76 \pm 8$ & $76 \pm 7$ & $0.003 \pm 0.048^{\mathrm{a}, \mathrm{b}}$ \\
Annual change in & & & $0.041 \pm 0.105^{\mathrm{a}}$ \\
AveIMT (mm/year) & $0.064 \pm 0.045$ & $0.032 \pm 0.036^{\mathrm{a}}$ & $0.044 \pm 0.106^{\mathrm{a}}$ \\
MaxIMT(mm/year) & $0.114 \pm 0.131$ & & \\
\hline
\end{tabular}

Data are shown as means \pm SD. Statistical significance was determined using one-way analysis of variance. T-chol, total cholesterol; TG, triglyceride; HDL-chol, HDL cholesterol; sBP, systolic blood pressure; dBP, diastolic blood pressure;
AveIMT, average IMT; MaxIMT, maximum IMT. a significant difference vs glibenclamide: $p<0.05$; ${ }^{\mathrm{b}}$ significant difference vs gliclazide

Table 3. Multivariate regression analysis for annual change in average IMT and maximum IMT

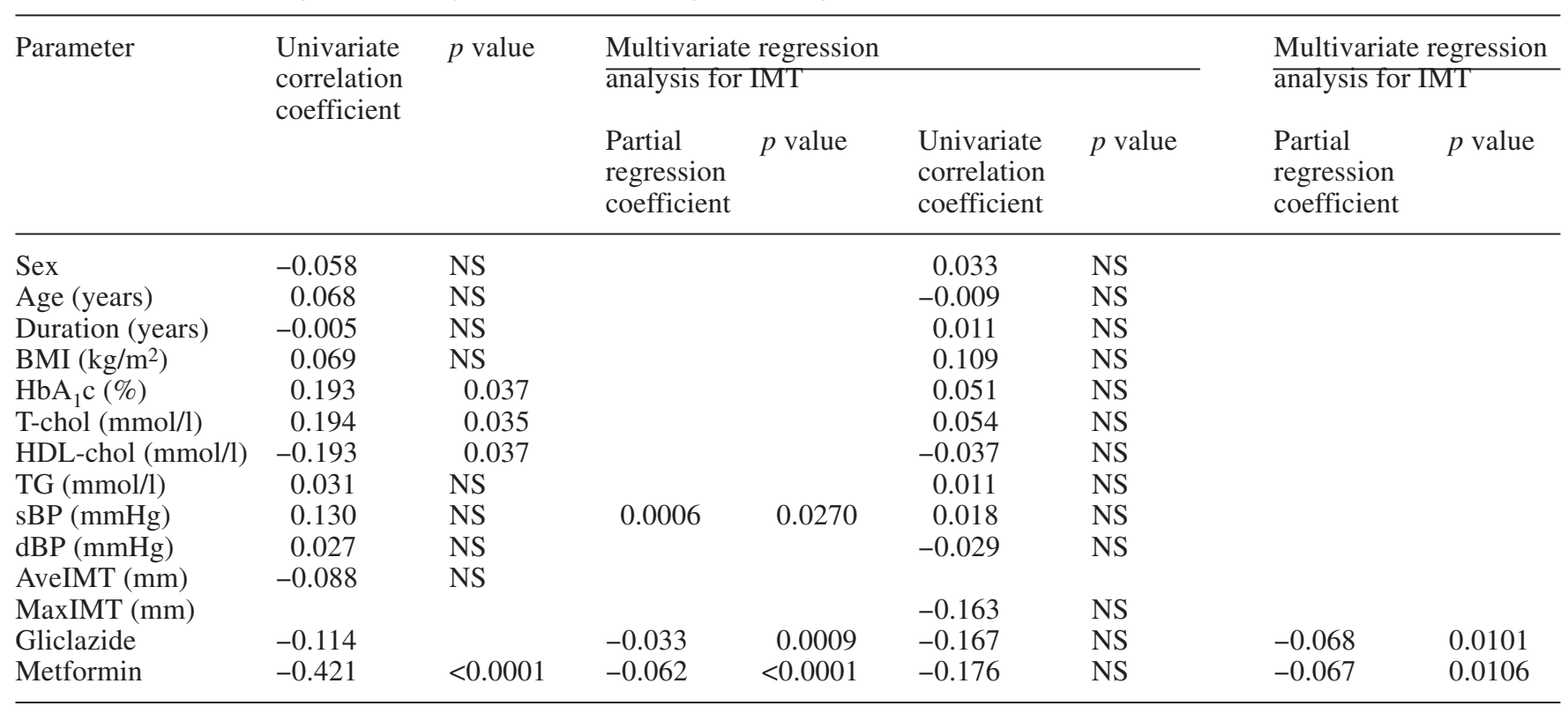

Stepwise multivariate regression analyses were performed to evaluate risk factors for the annual changes of AveIMT and maxIMT. Sex: men $=1$, women $=0$. T-chol, total cholesterol; TG, triglyceride; HDL-chol, HDL cholesterol; sBP, systolic

change in average IMT was smaller than that of the glibenclamide group $(p=0.005)$ (Table 2, Fig. 1). The annual progression of maximum IMT in the gliclazide group $(0.044 \pm 0.106 \mathrm{~mm})$ and the glibenclamide plus metformin group $(0.041 \pm 0.105 \mathrm{~mm})$ was smaller than that of the glibenclamide group $(0.114 \pm 0.131 \mathrm{~mm})$ (Table 2, Fig. 2). There was no significant difference in annual change of maximum IMT between the gliclazide group and the glibenclamide plus metformin group (Fig. 2). As a group, the annual change in average IMT showed a linear relationship with average haemoglobin $\mathrm{A}_{1} \mathrm{c} \quad(r=0.193$, blood pressure; dBP, diastolic blood pressure; AveIMT, average IMT; MaxIMT, maximum IMT. Gliclazide and metformin: administration $=1 ;$ no administration $=0$

$p=0.037) \quad$ (Fig. 2), total cholesterol $(r=0.194$, $p=0.035$ ) (Fig. 3), and HDL cholesterol concentrations $(r=-0.193, p=0.037)$ (Fig. 4). The annual change in average IMT showed no significant relationship with the change in haemoglobin $\mathrm{A}_{1} \mathrm{c}$ before and after the observation period.

Correlation between progression of carotid IMT and possible coronary risk factors. Stepwise multivariable regression analysis demonstrated that the administration of metformin (regression coefficient = $-0.062 \mathrm{~mm} /$ year, $p<0.0001$ ) and administration of gli- 


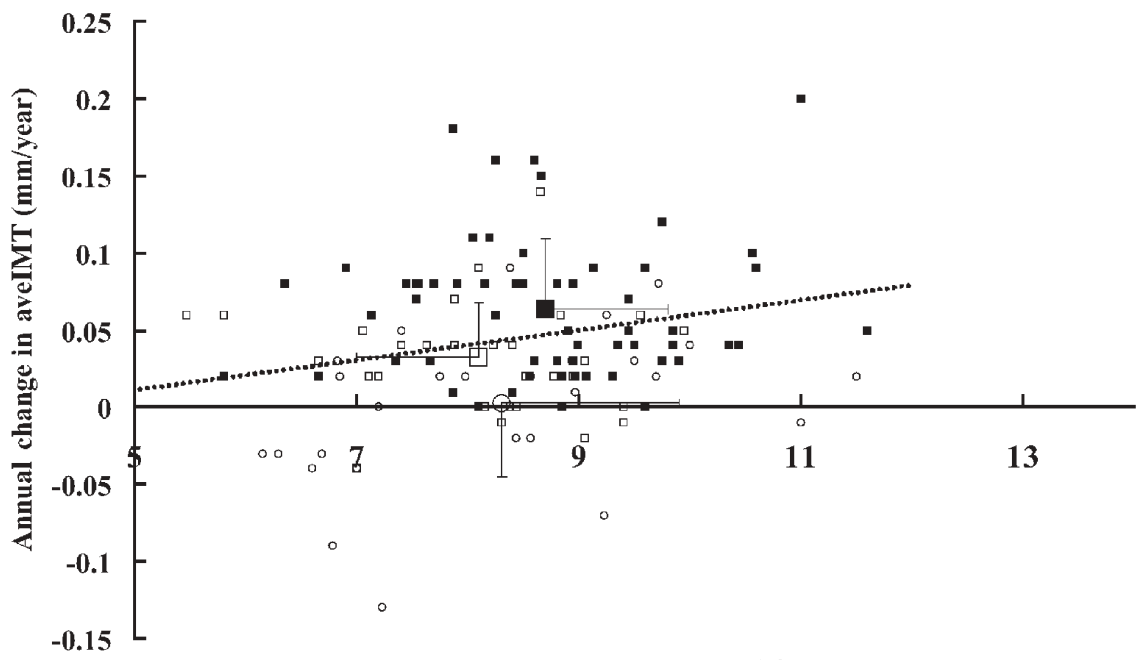

\section{Haemoglobin $A_{1} c(\%)$}

Fig. 1. Relationship between annual change in average IMT and haemoglobin $\mathrm{A}_{1} \mathrm{c}$ in the type 2 diabetic subjects treated with glibenclamide ( $\square$ ), gliclazide $(\square)$, and glibenclamide + metformin $(\bigcirc)$. A large closed square, a large open square, and a large open circle show mean values of annual change in average IMT (aveIMT) and haemoglobin $\mathrm{A}_{1} \mathrm{c}$ for each treatment group. Bars show SD of these values. The annual change in aveIMT of the glibenclamide plus metformin group $(0.003$ $\pm 0.048 \mathrm{~mm}$ ) was significantly smaller than that of the glibenclamide group $(0.064 \pm 0.045 \mathrm{~mm})$ and gliclazide group $(0.032 \pm 0.036 \mathrm{~mm})(p<0.0001$ and $p=0.043$ respectively $)$. In the gliclazide group, aveIMT increased during the follow-up period, but annual change in aveIMT was significantly smaller than that of the glibenclamide group $(p=0.005)$. As a group, annual change in aveIMT showed a linear relationship with average haemoglobin $\mathrm{A}_{1} \mathrm{c}(r=0.193, p=0.037)$ (broken line)

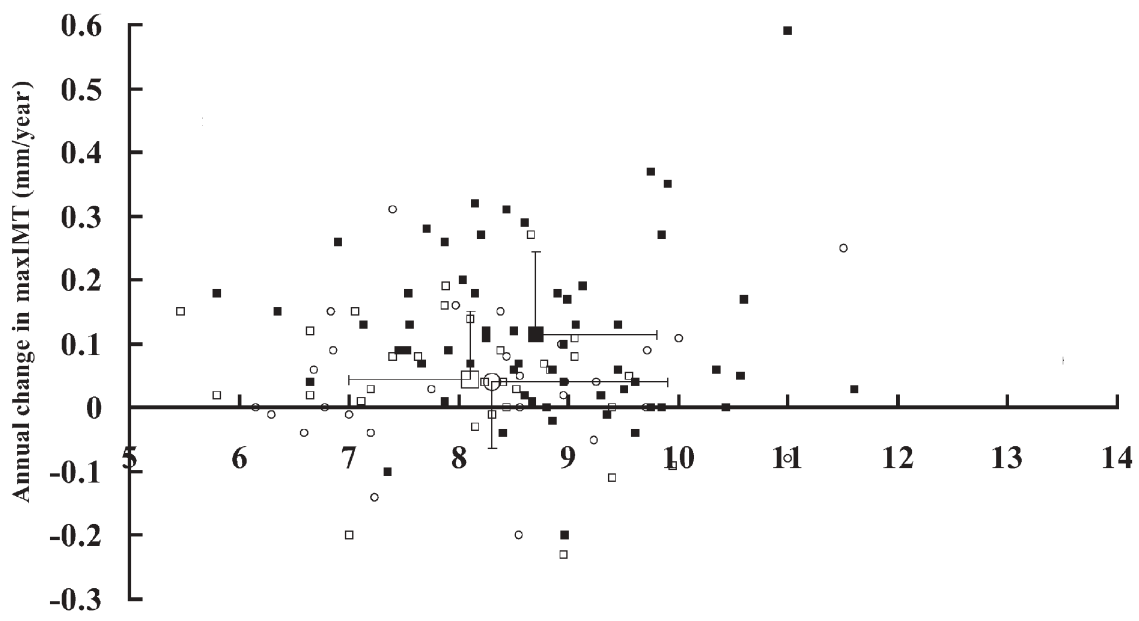

\section{Haemoglobin $\mathrm{A}_{1}$ c (\%)}

Fig. 2. Relationship between annual change in maximum IMT and haemoglobin $A_{1} c$ in the type 2 diabetic subjects treated with glibenclamide ( $\square$ ), gliclazide $(\square)$, and glibenclamide + metformin $(\bigcirc)$. A large closed square, a large open square, and a large open circle show mean values of annual change in maximum IMT (maxIMT) and haemoglobin $\mathrm{A}_{1} \mathrm{c}$ for each treatment group. Bars show the SD of these values. The annual progression in maxIMT of the gliclazide group $(0.044$ $\pm 0.106 \mathrm{~mm}$ ) and the glibenclamide plus metformin group $(0.041 \pm 0.105 \mathrm{~mm})$ was significantly smaller than that of the glibenclamide group $(0.114 \pm 0.131 \mathrm{~mm})(p=0.035$ and $p=0.029$ respectively). There was no significant difference in annual change in maxIMT between the gliclazide group and the glibenclamide plus metformin group. There was no significant linear relationship between annual change in maxIMT and haemogobin $\mathrm{A}_{1} \mathrm{c}$ clazide ( regression coefficient $=-0.033 \mathrm{~mm} / \mathrm{year}$, $p=0.0009$ ) independently decreased the annual change in average IMT and that systolic blood pressure $(p=0.027)$ was an independent risk factor for progression of average IMT (Table 3). The administration of metformin (regression coefficient $=-0.067 \mathrm{~mm} /$ year, $p=0.0106)$ and administration of gliclazide (regression coefficient $=-0.068 \mathrm{~mm} /$ year, $p=0.0101$ ) were shown to independently decrease the annual change in maximum IMT (Table 3). 


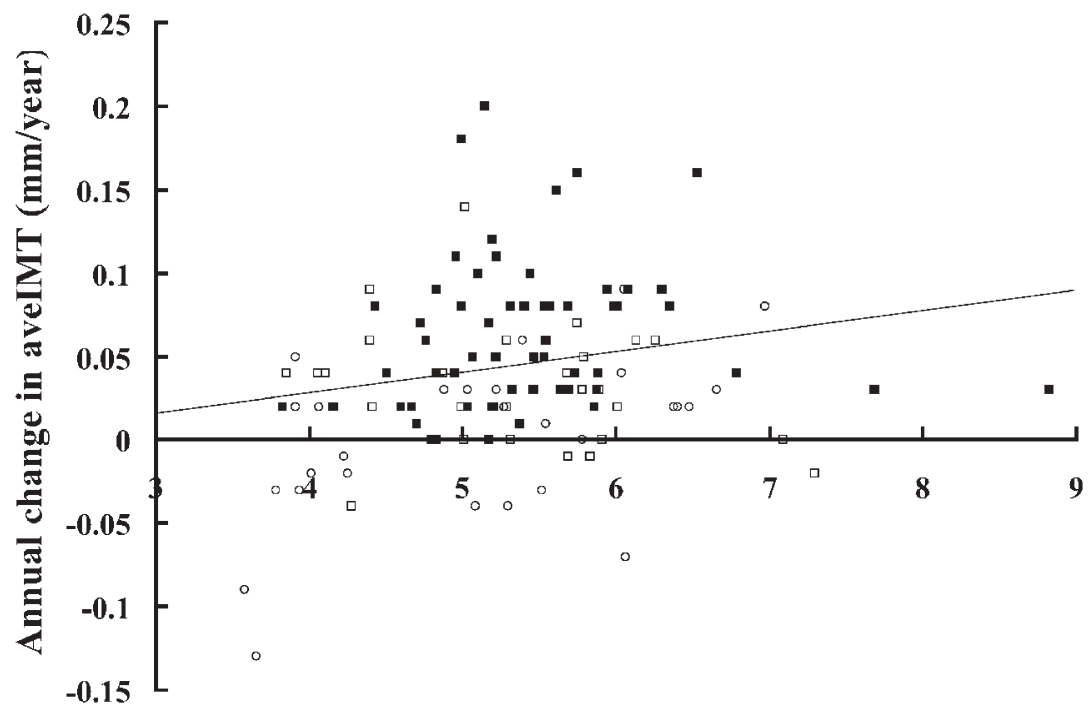

Total cholesterol ( $\mathrm{mmol} / \mathrm{l})$

Fig. 3. Relationship between annual change in average IMT and haemoglobin $\mathrm{A}_{1} \mathrm{c}$ in the type 2 diabetic subjects treated with glibenclamide ( $\square$ ), gliclazide $(\square)$, and glubenclamide
+ metformin $(\bigcirc)$. The solid line shows a significant linear relationship between annual change in average IMT (aveIMT) and total cholesterol $(r=0.194, p=0.035)$

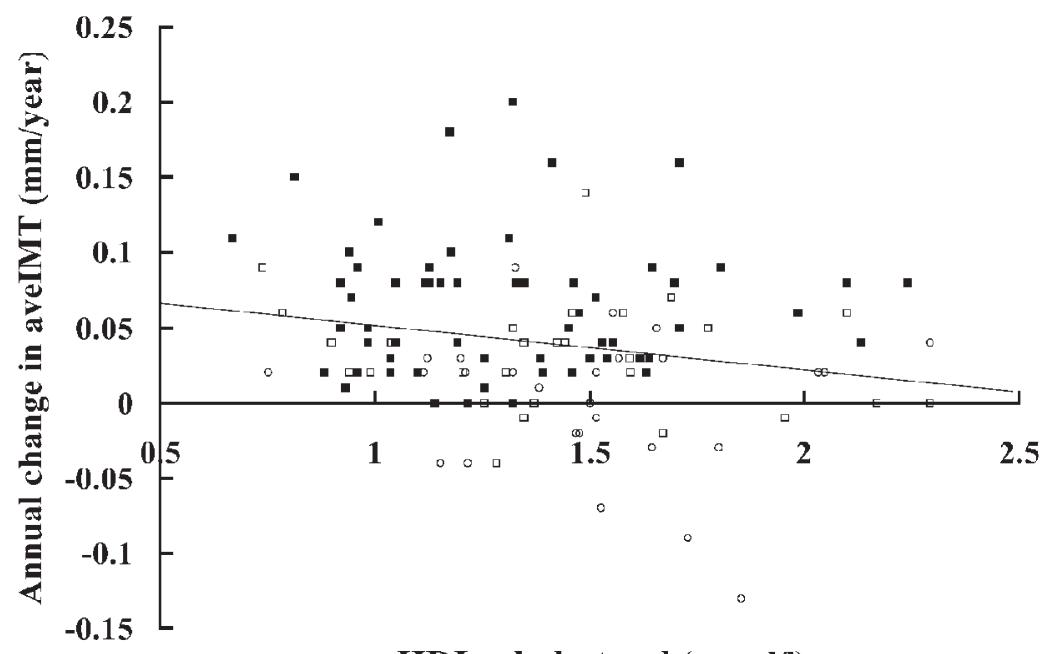

HDL-cholesterol (mmol/l)

Fig. 4. Relationship between annual change in average IMT and HDL-cholesterol in the type 2 diabetic subjects treated with glibenclamide $(\square)$, gliclazide $(\square)$, and glibenclamide + metformin $(\bigcirc)$. The solid line shows a significant linear relationship between annual change in average IMT (aveIMT)and HDL-cholesterol $(r=-0.193, p=0.037)$

\section{Discussion}

Effect of adding metformin to glibenclamide treatment on the progression of carotid artery IMT. To determine the effect on atherosclerosis in type 2 diabetes of adding metformin to sulphonylurea treatment, we examined the progression of carotid artery IMT as a surrogate end point. The progression of ca- rotid IMT was seen in subjects treated with glibenclamide or gliclazide alone, whereas it was almost totally inhibited in subjects treated with glibenclamide plus metformin. Metformin has been thought to provide some beneficial effects on the prevention of atherosclerosis, which may share common antecedents with diabetes [19]. First of all, metformin lowers blood glucose levels [20]. Next, it improves lipid profiles in type 2 diabetic patients, whether it is used with a sulphonylurea or not $[20,21,22,23,24$, 25]. In fact, in the present study, $\mathrm{HbA}_{1} \mathrm{c}$ levels in the glibenclamide plus metformin group significantly improved during the observation period. The lipid profiles were also improved but not significantly. Stepwise multivariable regression analysis indicated that administration of metformin independently accounts for the inhibition of the progression of carotid 
IMT, while these classical risk factors were included into the analysis. Therefore, the beneficial effect of metformin may be explained by other mechanisms. One possible mechanism by which metformin improves atherosclerosis is its anti-thrombotic effects $[26,27]$. The other possible mechanism is its ability to modulate reactive oxygen species generation [28]. The reduction of systemic methylglyoxal concentration induced by metformin may also have a beneficial effect on IMT [29].

Effect of gliclazide on the progression of carotid artery IMT. The progression of IMT was attenuated in the subjects treated with gliclazide as compared with the glibenclamide group. Although average $\mathrm{HbA}_{1} \mathrm{c}$ during the observation period was somewhat lower in the gliclazide than in the glibenclamide group, the difference was not significant. Stepwise multivariable regression analysis indicated that administration of gliclazide accounts for the attenuation of the progression of carotid IMT irrespective of $\mathrm{HbA}_{1} \mathrm{c}$. Gliclazide is a second-generation sulphonylurea that has been shown to be a free radical scavenger. Studies have shown that it provides beneficial effects on the haemorrheological abnormalities seen in diabetic vascular disease $[4,5,6,7,8,9]$. For example, gliclazide restores endothelial function [4] and reduces platelet reactivity [5]. Although not examined in this study, these actions may explain the beneficial effect of gliclazide on the IMT.

In conclusion, our results suggest that the administration of metformin plus glibenclamide or gliclazide, rather than glibenclamide alone, attenuates the progression of atherosclerosis in human carotid arteries in subjects with type 2 diabetes.

\section{References}

1. UK Prospective Diabetes Study UKPDS Group (1998) Effect of intensive blood glucose control with metformin on complications in overnight patients with type 2 diabetes (UKPDS 34). Lancet 352:854-865

2. West CI (2000) Radicals in oxidative stress in diabetes. Diabet Med 17:171-180

3. Scott NA, Jennings PE, Brown J, Belch JJ (1991) Gliclazide: a general free radical scavenger. Eur J Pharmacol 208:175-177

4. Pagano PJ, Griswold MC, Ravel D, Cohen RA (1998) Vascular action of the hypoglycaemic agent gliclazide in diabetic rabbits. Diabetologia 41:9-15

5. Jennings PE, Scott NA, Saniabadi AR, Belch JJ (1992) Effects of gliclazide on platelet reactivity and free radicals in type II diabetic patients: clinical assessment. Metabolism 41:36-39

6. Fu ZZ, Yan T, Chen YJ, Sang JQ (1992) Thromboxane/prostacyclin balance in type II diabetes: gliclazide effects. Metabolism 41 [Suppl 1]:33-35

7. Gram J, Kold A, Jespersen J (1989) Rise of plasma t-PA fibrinolytic activity in a group of maturity onset diabetic pa- tients shifted from a first generation (tolubutamide) to a second generation sulfonylurea (gliclazide). J Intern Med 225:241-247

8. Renier G, Desfaits A-C, Serri O (2000) Gliclazide decreases low-density lipoprotein oxidation and monocyte adhesion to the endothelium. Metabolism 49 [Suppl]:1722

9. Renier G, Desfaits A-C, Serri O (2000) Effect of gliclazide on monocyte-endothelium interactions in diabetes. J Diabetes Complications 14:215-223

10. Kawamori R, Yamasaki Y, Matsushima H et al. (1992) Prevalence of carotid atherosclerosis in diabetic patients. Ultrasound high-resolution B-mode imaging on carotid arteries. Diabetes Care 15:1290-1294

11. O'Leary DH, Polak JF, Kronmal RA et al. (1992) Distribution and correlates of sonographically detected carotid artery disease in the Cardiovascular Health Study. Stroke 23:1752-1760

12. Bots ML, Hoes AW, Koudstaal PJ, Hofman A, Grobbee DE (1997) Common carotid intima-media thickness and risk of stroke and myocardial infarction. Circulation 96:14321437

13. Yamasaki Y, Kodama M, Nishizawa H et al. (2000) Carotid intima-media thickness in Japanese type 2 diabetic subjects: predictors of progression and relationship with incident coronary heart disease. Diabetes Care 23:13101315

14. Shinoda-Tagawa T, Yamasaki Y, Yoshida S et al. (2002) A phosphodiesterase inhibitor, cilostazol, prevents the onset of silent brain infarction in Japanese subjects with type II diabetes. Diabetologia 45:188-194

15. Ambrose JA, Tannenbaum MA, Alexopoulos D et al. (1998) Angiographic progression of coronary artery disease and the development of myocardial infarction. J Am Coll Cardiol 12:56-62

16. Yamasaki Y, Kawamori R, Matsushima H et al. (1994) Atherosclerosis in carotid artery of young IDDM patients monitored by ultrasound high-resolution B-mode imaging. Diabetes 43:634-639

17. Handa N, Matsumoto M, Maeda H et al. (1990) Ultrasonic evaluation of early carotid atherosclerosis. Stroke 21:15671572

18. Pignoli P, Tremoli E, Poli A, Oreste P, Paoletti R (1986) Intimal plus medial thickness of the arterial wall: a direct measurement with ultrasound imaging. Circulation 74: 1399-1406

19. Stern MP (1995) Diabetes and cardiovascular disease: the "common soil" hypothesis. Diabetes 44:369-374

20. Bailey CJ, Turner RC (1996) Metformin. N Engl J Med 334:574-579

21. Jeppesen J, Zhou MY, Chen YD, Reaven GM (1994) Effect of metformin on postprandial lipemia in patients with fairly to poorly controlled NIDDM. Diabetes Care 17:10931099

22. DeFronzo RA, Goodman AM (1995) Efficacy of metformin in patients with non-insulin-dependent diabetes mellitus. The Multicenter Metformin Study Group. N Engl J Med 333:541-549

23. DeFronzo RA, Barzilai N, Simonson DC (1991) Mechanism of metformin action in obese and lean noninsulin-dependent diabetic subjects. J Clin Endocrinol Metab 73: 1294-1301

24. Reaven GM, Johnston P, Hollenbeck CB et al. (1992) Combined metformin-sulfonylurea treatment of patients with noninsulin-dependent diabetes in fair to poor glycemic control. J Clin Endocrinol Metab 74:10201026 
25. Perriello G, Misericordia P, Volpi E et al. (1994) Acute antihyperglycemic mechanisms of metformin in NIDDM: evidence for suppression of lipid oxidation and hepatic glucose production. Diabetes 43:920-928

26. Nagi DK, Yudkin JS (1993) Effects of metformin on insulin resistance, risk factor for cardiovascular disease, and plasminogen activator inhibitor in NIDDM subjects: a study of two ethnic groups. Diabetes Care 16:621-629

27. Sobel BE (1999) Insulin resistance and thrombosis: a cardiologist's view. Am J Cardiol 84:37J-41J
28. Bonnefont-Rousselot D, Raji B, Walrand S et al. (2003) An intracellular modulation of free radical production could contribute to the beneficial effects of metformin towards oxidative stress. Metabolism 52:586-589

29. Thornalley PJ (1996) Pharmacology of methylglyoxal: formation, modification of proteins and nucleic acids, and enzymatic detoxification - a role in pathogenesis and antiproliferative chemotherapy. Gen Pharmcol 27:565-573 